\title{
Dural Tear: A Feared Complication of Lumbar Discectomy
}

\author{
Serdal ALBAYRAK ${ }^{1}$, Sait OZTURK², Omer AYDEN ${ }^{1}$, Necati UCLER ${ }^{3}$ \\ ${ }^{1}$ Elazig Education and Research Hospital, Department of Neurosurgery, Elazig, Turkey \\ ${ }^{2}$ Firat University, School of Medicine, Department of Neurosurgery, Elazig, Turkey \\ ${ }^{3}$ Adiyaman University, Education and Research Hospital, Department of Neurosurgery, Adiyaman, Turkey
}

\section{ABSTRACT}

AIM: To show causes of dural tear in isolated lumbar disc surgery, and to investigate the risk factors.

MATERIAL and METHODS: We retrospectively reviewed 1159 cases (532 females and 627 males) involving patients who underwent a surgery for the treatment of lumbar disc herniation between 2006 and 2013 . We have analysed the side of the surgery, level of the operation, first or revision surgery, type of anesthesia and type of surgical procedure for the risk of dural tear. To examine differences between disc levels, we used Chi-square testing for categorical variables and the student's t test for continuous variables. To analyze our data, we used STATA version 12. A "p value" less than 0.05 was considered as statistically significant.

RESULTS: A total of 1047 (90.3\%) cases were treated with microdiscectomy, and $112(9.7 \%)$ required open discectomy. Overall, $820(70.7 \%)$ and $339(29.3 \%)$ surgeries were performed under epidural and general anesthesia, respectively. Dural tear ratio was $1.20 \%$. In dural tear ratio, there was a significant difference in gender (Female: $1.6 \%$, Male: $0.79 \%)(p<0.05)$. Dural tear ratios at primary disc surgery and at recurrent disc surgery were respectively $0.82 \%$ and $7.14 \%(p<0.05)$. Most of the tears were on the right side $(11 / 14)(p<0.05) .13$ dural tear cases $(1.58 \%)$ were noted in patients who operated under epidural anesthesia $(820$ cases) compared to $1(0.29 \%)$ under general anesthesia (339 cases) $(p<0.05)$.

CONCLUSION: Recurrent disc surgery, female sex, epidural anesthesia, open discectomy, non-dominant hand usage of surgeon, and upper-level affected lumbar discs were risk factors for intraoperative dural tear during lumbar disc surgery.

KEYWORDS: Dural tear, Epidural anesthesia, Lumbar discectomy, Recurrent disc

\section{INTRODUCTION}

$\mathrm{D}$ ural tear (DT) during spinal surgery is an expected but feared complication. Intraoperative DT can result in lengthened hospital stays and increased rates of infectious complications (7). Lumbar disc surgery is considered an elective surgery (except in cases of cauda equina syndrome), and intraoperative DT may be considered by both patients and surgeons as a surgical failure. Expected complications of DT include cerebrospinal fluid (CSF) leakage, and this can lead to pseudomeningocele, dura-cutaneous fistula, meningitis, arachnoiditis, epidural abscess, intracranial subdural hematoma, nerve root entrapment, wound healing complications, persistent headache, or reoperation for leak repair (7).
The incidence of DT has been reported to be $0.5 \%-18 \%$ (1-3, $7,10)$. Risk factors for this complication include the surgeon's experience and patient's age (4), but some other factors may also be important and should be specifically investigated.

This study aimed to describe the risk factors and clinical outcomes of DT during microdiscectomy among 1159 operated patients who underwent lumbar disc surgery.

\section{MATERIAL and METHODS}

We retrospectively reviewed 1159 consecutive cases involving patients who underwent a surgical procedure for the treatment of lumbar disc herniation between 2006 and 2013. Each 
surgeon had more than 6 years of experience with spine surgery, and all were right-handed. After we excluded patients with spinal tumor or trauma, our sample of 1159 patients included 532 females and 627 males with a mean age of 49.6 years (range 17-82 years) at the time of surgery (Table I). Classification of herniated discs which defined by Fardon et al. was used (5).

The lumbar disc distributions were: 1041 patients with 1 involved level, 97 patients with 2 involved levels, 15 patients with a single bilateral level, 2 patients requiring total laminectomy, and 4 patients with 3 involved levels. Revision surgery was performed in $70(6 \%)$ patients, and for these cases, 22 of the first surgeries had been performed by our department. A total of $1047(90.3 \%)$ cases were treated with microdiscectomy, and $112(9.7 \%)$ required open discectomy. Overall, 820 (70.7\%) and $339(29.3 \%)$ surgeries were performed under epidural and general anesthesia, respectively (Table I).

For DT (14 of 1159), we performed primary repairs with 6-0 nylon sutures and covered the area with fibrin glue. In one case with small DTs, fibrin glue was simply sprayed on without suturing performed, and although the last DT was not directly seen, CSF was observed and fibrin glue was simply sprayed on the area. No nerve injury was identified in the 14 patients with DT.

Among the 14 DT patients, 12 and 2 were mobilized on the first and second postoperative day, respectively. All patients with DT received dual antibiotic and acetazolamide treatment in the postoperative period.
Mean follow-up time after surgery is 26 months (Min: 14, Max: 53).

To examine differences between disc levels, we used Chisquare testing for categorical variables and the Student $t$ test for continuous variables. To analyze our data, we used STATA version 12. A p value less than 0.05 was considered statistically significant.

\section{RESULTS}

The most frequently affected level was $L_{4-5}$ (590 cases: 308 left-sided, 282 right-sided) followed by $L_{5}-S_{1}$ (390 cases: 204 left-sided, 186 righ-sided), $L_{3-4}$ (50 case: 27 left-sided, 23 rightsided), $L_{2-3}$ (4 left-sided, 2 right-sided), and $L_{1-2}$ ( 3 left-sided, 2 right-sided) (Table I). Overall, 525 patients had extruded lumbar disc herniations, 283 had sequestrated herniations, 300 had protrusions, and 51 had lumbar stenosis and lateral recess obliteration (Table I).

Among the 1159 patients, 14 (1.2\%) had DTs, including 9 females and 5 males. The mean ages at the time of surgery for patients with and without DT were 49.6 and 40.5 years, respectively, and this difference was statistically significant $(p<0.05)$. There was also a significant difference in the incidence of DT based on sex (9/532 females vs. 5/627 males) $(p<0.05)$ (Table II).

Among the 14 cases with DTs, 8 were on the right side of the $L_{4-5}$ level (4 cases with protrusion, 2 with recurrent disc, and 2 with extruded), 2 were on the right $L_{5}-S_{1}$ level ( 1 with protrusion and 1 with recurrent), 2 cases (protrusion) were on

Table I: Patient Demographic and Clinical Characteristics

\begin{tabular}{|c|c|c|c|c|}
\hline & & n (\%) & n (\%) & Total \\
\hline \multirow{2}{*}{ Gender } & & Female & Male & \\
\hline & & $522-(45.9)$ & $627-(54.1)$ & 1159 \\
\hline \multirow{2}{*}{ Type of Surgery } & & Microdiscectomy & Open Discectomy & \\
\hline & & $1047(90.3)$ & $112(9.7)$ & 1159 \\
\hline \multirow{2}{*}{ Type of Anesthesia } & & Epidural & General & \\
\hline & & $820(70.7)$ & $339(29.3)$ & 1159 \\
\hline \multirow[t]{2}{*}{ Type of Disc (n) } & Extruded & Sequestrated & Protrusion & LS + LRO \\
\hline & 525 & 283 & 300 & 51 \\
\hline \multirow{6}{*}{ Operated Levels } & & Right (n) & Left (n) & Total \\
\hline & L1-L2 & 2 & 3 & 5 \\
\hline & L2-L3 & 2 & 4 & 6 \\
\hline & L3-L4 & 23 & 27 & 50 \\
\hline & L4-L5 & 282 & 308 & 590 \\
\hline & L5-S1 & 186 & 204 & 390 \\
\hline
\end{tabular}

n: Number, \%: Percent, LS: Lumbar stenosis, LRO: Lateral recess obliteration. 
the left side, 1 case (recurrence) was at the right $\mathrm{L}_{3-4}$ level, and 1 patient had a recurrent lumbar disc on the left $L_{5}-S_{1}$ level. We observed 5 DTs in 70 patients $(7.14 \%)$ with recurrent disc herniation. 8 (1.41\%) DTs in 590 cases with $\mathrm{L}_{4-5}$ disc herniation, 3 DTs in 390 cases with $L_{5}-S_{1}$ level, and 3 (15\%) DTs in 50 cases with $\mathrm{L}_{3-4}$ levels (Table II).

The number of total recurrent discs was 70 , and the DT ratio in recurrent disc patients was $7.14 \%(p<0.05)$. The DT rate in patients without recurrent disc surgery was $0.82 \%(p<0.05)$. DT was most commonly seen on the right side of the $L_{4-5}$ level $(8 / 282$ patients, $2.83 \%)$ but $3 / 27(11.1 \%)$ patients who underwent left side $L_{3-4}$ surgery also had DTs $(p<0.05)$. All DTs occurred in patients that operated for single level.

In cases of midline protrusion and ligamentum flavum incision with a Kerrison rongeur, DTs occurred in 8/14 patients despite pad use. In 5/14 patients, DTs affect the upper part of the lower lamina after flavectomy. Interestingly, no DTs occurred when the ligamentum flavum was raised with an elevator and then cut with lancet. Most DTs (13/14) occurred during flavectomy with a Kerrison rongeur $(p<0.05) ; 1$ occurred during retraction, and the other 13 were noted during recurrent fragment removal and CSF was seen but dural defects were not detected. During 70 reoperations, we saw 5 DTs (1 during root retraction, 1 during fragment pulling, and 3 during the excision of granulation tissue).

\section{DISCUSSION}

DT is one of the most frequent serious complications of spinal surgery, and despite effective treatment modalities, it is generally feared by surgeons. DTs have been looked at in the literature before (1-3).

The incidence of DT has been reported to be $0.5 \%-18 \%$ (1$3,7,8)$; we calculated an incidence of $1.2 \%$. When patients with recurrent disc disease were excluded from the study, the incidence decreased to $0.82 \%$. In patients with recurrent disc disease, the incidence was $7.14 \%$. This wide range is probably because incidence rates in multicenter studies have been estimated using questionnaires alone rather than prospective data (8). Cammisa et al. and Takahashi et al. reported that patients without DT were younger than those with DT $(2,9)$. We obtained similar results in our study (average ages of 40.5 and 49.6 years, respectively). Similar to Takahashi et al. (9), we also observed a higher incidence of DT in females compared to $(9 / 532$ vs. $5 / 627$, respectively).

We observed that surgery to correct recurrent disc herniation had a higher DT rate, presumably because of the anatomical complexity, adhesions, and scar tissue due to previous operation(s). The majority of DTs occur during spinal surgery, especially in revision/secondary procedures. Bosacco et al. retrospectively assessed the incidence of traumatic durotomy occurring during 2,144 spinal operations performed over a 10-year period (followed for 22.4 months) and found that $66(3.1 \%)$ had traumatic dural fistulas that most frequently occurred during revision surgery (1). Of interest, 60 fistulas were discovered intraoperatively and were directly repaired, while $6(0.28 \%)$ fistulas not recognized during the first surgery (5 with pseudomeningoceles) failed to respond to conservative measures, and required additional surgery. In our study, we observed CSF leakage in one patient, and there were no instances of pseudomeningocele after surgery.

We mostly operated on patients under epidural anesthesia. Notably, 13 DTs (1.58\%) were noted in patients who received epidural anesthesia (820 cases) compared to $1(0.29 \%)$ under general anesthesia (339 cases) $(p<0.05)$. Obviously when performing lumbar disc surgery under epidural anesthesia, the dural sac is mobile, especially during breathing. In addition, patients can move in response to pain during medial

Table II: DT Distribution by Sex, Age, Disc Side, and Surgery Type

\begin{tabular}{|c|c|c|c|c|}
\hline & & & Total & $\mathbf{p}$ \\
\hline \multirow{2}{*}{ Gender (n) } & Female & Male & & \\
\hline & 9 & 5 & 14 & 0.089 \\
\hline \multirow{2}{*}{ Age at Time of Surgery (years) } & with DT & without DT & & \\
\hline & 49.6 & 40.5 & & 0.043 \\
\hline \multirow{2}{*}{ Side of DT } & Right & Left & & \\
\hline & 11 & 3 & & 0.039 \\
\hline DT and Disc Levels in Primary Surgery & $n-\%$ & & & \\
\hline L3-L4 & $3 / 50(15 \%)$ & & & 0.045 \\
\hline L4-L5 & $8 / 590(1.41 \%)$ & & & 0.043 \\
\hline L5-S1 & $3 / 390(0.76 \%)$ & & & 0.036 \\
\hline \multirow[t]{2}{*}{ DT in Revision Surgery } & $n-\%$ & & & \\
\hline & $5 / 70(7.14 \%)$ & & & 0.037 \\
\hline
\end{tabular}

n: Number, \%: Percent, DT: Dural tear. 
facetectomy or to cough, sneeze, or strain, so surgeons are more likely to encounter DTs compared to during general anesthesia.

Although DTs were most commonly seen at the $L_{4-5}$ level, the $L_{3-4}$ level had a higher DT rate, especially on left side. We thought that this might be due to the narrower spinal canal diameter in the upper lumbar region, short lamina length, and decreased maneuvering capacity for surgeons at the $L_{3-4}$ level. In addition, Hong et al. assessed dura mater thickness with respect to sex and age in more than 300 cadaveric specimens at each level between $T_{1-2}$ and $L_{5}-S_{1}(6)$. The thinnest portion of the dura mater was at $L_{2-3}$ and $L_{3-4}$. They also reported that dural sac thickness increased with age, but there was no difference between sexes, which corroborates our DT rates, especially for higher lumbar discs.

It is important to note that, most of the DTs were on the right side (11 vs. $3, p<0.05)$. All of the surgeons in this study were right handed, but surgeries for right-sided lumbar discs required them to use their non-dominant or in other words non-practical hand. Therefore, non-dominant hand usage of surgeons can be accepted as a risk factor for DT.

\section{- CONCLUSION}

Overall, recurrent disc surgery, female sex, epidural anesthesia, open discectomy, non-dominant hand usage of surgeon, and upper-level affected lumbar discs are risk factors for intraoperative DTs. Midline disc localization and cutting of the ligamentum flavum with Kerrison rongeur may also predispose patients to DT development. When used carefully, employing a scalpel to cut the ligamentum flavum may not increase the risk of dural injury. Although significant, our results should be confirmed in multicenter studies that assess DTs in patients with other pathologies besides lumbar disc disease.

\section{REFERENCES}

1. Bosacco SJ, Gardner MJ, Guille JT: Evaluation and treatment of dural tears in lumbar spine surgery: A review. Clin Orthop Relat Res 389: 238-247, 2001

2. Cammisa FP Jr, Girardi FP, Sangani PK, Parvataneni HK, Cadag $S$, Sandhu HS: Incidental durotomy in spine surgery. Spine 25: 2663-2667, 2000

3. Epstein NE: The frequency and etiology of intraoperative dural tears in 110 predominantly geriatric patients undergoing multilevel laminectomy with noninstrumented fusions. J Spinal Disord Tech 20: 380-386, 2007

4. Espiritu MT, Rhyne A, Darden BV II: Dural tears in spine surgery. J Am Acad Orthop Surg 18: 537-545, 2010

5. Fardon DF, Williams AL, Dohring EJ, Murtagh FR, Gabriel Rothman SL, Sze GK: Lumbar disc nomenclature: Version 2.0: Recommendations of the combined task forces of the North American Spine Society, the American Society of Spine Radiology and the American Society of Neuroradiology. Spine J 14: 25252545, 2014

6. Hong JY, Suh SW, Park SY, Modi HN, Rhyu IJ, Kwon S, Yu H, Byun J: Analysis of dural sac thickness in human spine-cadaver study with confocal infrared laser microscope. Spine J 11:11211127, 2011

7. Luszczyk MJ, Blaisdell GY, Wiater BP, Bellabarba C, Chapman JR, Agel JA, Bransford RJ: Traumatic dural tears: What do we know and are they a problem? Spine J 14: 49-56, 2014

8. Tafazal SI, Sell PJ: Incidental durotomy in lumbar spine surgery: Incidence and management. Eur Spine J 14: 287-290, 2005

9. Takahashi Y, Sato T, Hyodo H, Kawamata T, Takahashi E, Miyatake $\mathrm{N}$, Tokunaga $\mathrm{M}$ : Incidental durotomy during lumbar spine surgery: Risk factors and anatomic locations. J Neurosurg Spine 18:165169, 2013

10. Wang JC, Bohlman HH, Riew KD: Dural tears secondary to operations on the lumbar spine. Management and results after a two-year-minimum follow-up of eighty-eight patients. J Bone Joint Surg Am 80: 1728-1732, 1998 